\title{
PROPORTION OF NEONATAL SEPSIS AMONG STUDY SUBJECTS WITH OR WITHOUT JAUNDICE IN A TERTIARY CARE CENTRE OF AGARTALA, TRIPURA
}

\author{
Sanjib Kumar Debbarma1, Tapan Majumdar², Sujit Kumar Chakrabarti³, Nazrul Islam ${ }^{4}$ \\ ${ }^{1}$ Associate Professor, Department of Paediatrics, Agartala Government Medical College. \\ ${ }^{2}$ Associate Professor, Department of Microbiology, Agartala Government Medical College. \\ ${ }^{3}$ Assistant Professor, Department of Paediatrics, Agartala Government Medical College. \\ 4Junior Resident, Department of Paediatrics, Agartala Government Medical College.
}

\section{ABSTRACT}

\section{BACKGROUND}

Neonatal sepsis remains a major cause for neonatal mortality and morbidity. Approximately, 1 million deaths per year are caused by infection occurring in the neonatal period, accounting for over $25 \%$ of global neonatal deaths. In India, it is about $19 \%$.

The aim of the present study was to find out the prevalence and clinico-aetiological profile of Neonatal Sepsis with objectives: i) To determine the relationship of CRP, IL- 6 and pro-calcitonin level with blood culture, ii) To evaluate whether these markers have any relationship on the outcome of these infections.

\section{MATERIALS AND METHODS}

Blood was collected from suspected neonates. Total leucocyte count, immature/total neutrophils ratio, ANC, micro ESR, CRP and serum bilirubin was estimated. Blood culture was done to isolate and identify the organism using standard protocol. Antibiogram of the isolated organism was carried out by disc diffusion method. IL-6 and serum pro-calcitonin (PCT) estimation was performed only in culture positive cases.

\section{RESULTS}

Among 170 suspected cases, $42.4 \%$ had early onset sepsis and $57.6 \%$ had late onset sepsis. Sepsis developed in $60 \%$ neonates whose mother had history of PROM; other important risk factors was low birth weight. Blood culture was positive in $36.5 \%$ cases. Out of all blood culture positive cases $64.5 \%$ had IL-6 $\geq 20 \mathrm{pg} / \mathrm{mL}$ and procalcitonin $\geq 10 \mathrm{pg} / \mathrm{mL}$ in $95.1 \%$ cases. Bacteriological profile showed Klebsiella spp. as most predominant organism followed by Staphylococcus aureus. Antibiotic susceptibility pattern showed high degree of resistance towards penicillins, cephalosporins, fluoroquinolones and less resistance was observed in aminoglycosides, imipenem, vancomycin and linezolid. Among 62 confirmed neonatal sepsis cases, mortality was found around $42 \%$.

\section{CONCLUSION}

Neonatal sepsis was found to be high with presence of resistant phenotypes. Detection of acute phase reactants like CRP, IL- 6 and procalcitonin level helps in initiating appropriate treatment at the earliest where blood culture facilities are not available.

\section{KEYWORDS}

Neonatal Sepsis, Low Birth Weight, Procalcitonin, IL-6, Resistance Phenotypes.

HOW TO CITE THIS ARTICLE: Debbarma SK, Majumdar T, Chakrabarti SK, et al. Proportion of neonatal sepsis among study subjects with or without jaundice in a tertiary care centre of Agartala, Tripura. J. Evolution Med. Dent. Sci. 2016;5(97):7141-7145, DOI: $10.14260 /$ jemds/2016/1616

\section{BACKGROUND}

Neonatal sepsis is defined as a clinical syndrome of bacteraemia with systemic signs and symptoms of infection in the first 4 weeks of life. Approximately, 1 million deaths per year are caused by infection occurring in the neonatal period ( 0 - 28 days), accounting for over $25 \%$ of global neonatal deaths; $99 \%$ of these deaths occur in developing countries.[1]

Neonatal sepsis is broadly classified into early-onset sepsis ( $<72$ hours) and late onset sepsis ( $>72$ hours).

Financial or Other, Competing Interest: None.

Submission 28-10-2016, Peer Review 21-11-2016,

Acceptance 27-11-2016, Published 05-12-2016.

Corresponding Author:

Dr. Sanjib Kumar Debbarma

Associate Professor

Department of Paediatrics,

Agartala Government Medical College,

Agartala-799006, Tripura.

E-mail:dr sanjibdb@rediffmail.com

DOI: $10.14260 /$ jemds/2016/1616
Early onset sepsis (EOS) often presents as a fulminant, multi-system illness within 72 hours of delivery and is mainly due to bacteria acquired before and during delivery, whereas Late Onset Sepsis (LOS) is due to bacteria acquired after delivery (Nosocomial or community sources) and can present as either a fulminant or a smouldering infection. EOS presents with prominent respiratory signs, while LOS has more varied presentations. ${ }^{[2]}$

The neonatal mortality rate according to the data from National Neonatal Perinatal Database (NNPD, 2002-03) is 44 per 1000 live births and sepsis found to be one of the commonest causes of neonatal mortality contributing to $19 \%$ of all neonatal deaths. Sepsis related mortality is largely preventable with prevention of sepsis itself, timely recognition, rational antimicrobial therapy and aggressive supportive care. $[3,4,5]$

Clinical diagnosis of neonatal sepsis on the basis of the symptoms is very difficult.[6] Isolation of the causative microorganisms by using blood culture has been the gold standard for its diagnosis.[7] Despite the availability of effective antibiotics, early diagnosis represents a major 
challenge because of the non-specific nature of signs and symptoms and non-availability of standard microbial culture results in the first $48 \mathrm{~h}$. Rapid competent diagnostic markers of neonatal sepsis to make a clear distinction between neonates who have clinical signs because of sepsis were studied. Examples of these markers, which have been investigated with variable sensitivities and specificities, include Interleukin-6 (IL-6), Tumour Necrosis Factor (TNF), Procalcitonin (PCT), C-Reactive Protein (CRP), immunoglobulins and others. ${ }^{[8,9]}$

Procalcitonin (PCT), is detectable in plasma as early as 2 hours after the exposure the bacterial products, its level rises for 6 to 8 hours, reaches a plateau after $12 \mathrm{hrs}$., and then decreases to a normal level after 2 to 3 days.[10,11]

Recently, PCT has received an important role in the diagnosis of bacterial infection in both paediatric and adult population; however, its accuracy in neonatal early onset sepsis is still an issue of controversy.[12] Other acute phase reactants and interleukins are also used in the diagnosis of bacterial infection in the neonates. Among these, IL- 6 is the most well-known marker.[13,14,15,16]

CRP is one of the acute phase proteins. Although, it is a classical and sensitive marker of inflammation; it cannot be used to differentiate between bacterial and other infection. It is a disadvantage that CRP increases after PCT. This is why several authors have opined that it is important to be cautious with the interpretation of CRP values in children with fever lasting less than 12 hours, because at that time it may remain negative, although there is presence of sepsis.[17] Non-infectious condition as perinatal asphyxia, respiratory distress syndrome, brain haemorrhage and meconium aspiration syndrome and post-surgical period can induce abnormal values of CRP.[18] In contrast localised bacterial infections, severe viral infections and inflammatory reactions of non-infectious origin do not or only slightly increase PCT level.[19] The increase of PCT has been observed before the rise in CRP. The unique feature that PCT levels increase in bacterial and fungal infections, but remain unchanged even in severe viral infections and other inflammatory diseases makes PCT attractive as a potential diagnostic variable for the diagnosis of bacterial infection.[19]

Direct measurement of serum level of IL-6 and procalcitonin could make the earlier detection of infection possible compared to the measurement of acute phase reactant which in turn are secreted in response to the proinflammatory cytokines. Hence, our study aimed at assessing simultaneous measurement of PCT and IL-6 with blood culture.

\section{MATERIALS AND METHODS}

This was a two years hospital-based cross-sectional study. Neonates admitted in NICU during the study period with following Inclusion Criteria were taken up for the study.

1. Neonates with clinical signs and symptoms suggestive of sepsis (poor feeding, respiratory distress, fever and hypothermia, etc.).

2. Neonates whose mother have risk factors like history of prolonged rupture of membranes ( $>24 \mathrm{hrs}$.), maternal fever (Within 2 weeks prior to delivery, premature labour or instrumental delivery.

\section{Exclusion Criteria}

1. Neonates who were on antibiotics therapy.

2. More than 28 days of life.

\section{Collection of Sample}

Samples were collected from study subjects after obtaining written consent of the parents.

\section{Storage and Transportation of Sample}

Samples - Blood/serum was collected from neonates, clinically suspected to have features of septicaemia who were admitted in this hospital. The samples were transported to the laboratory immediately after collection. For serological tests, serum separated and kept in the refrigerator at $2-8^{0} \mathrm{C}$ on the same day.

- Blood Culture - 1-2 mL of venous blood was obtained for blood culture using Brain Heart Infusion broth and incubated at $37^{\circ} \mathrm{C}$ temperature. Subcultures were done on 2nd, 3rd, 5th and 7th days. Those subcultures, which were showing presence of bacterial colony were isolated and identified using the standard biochemical test.

- Antibiogram of the isolated organism was carried out by disc diffusion method as per CLSI guideline.

- Total Leucocyte Count (TLC), differential count and Absolute Neutrophil Count (ANC) were carried out by automated cell counter.

- C-Reactive Protein (CRP) estimation was done by Latex Agglutination test.

- Microerythrocyte Sedimentation Rate (ESR) was performed by Natelson heparinised capillary tubes.

- IL-6 and serum pro-calcitonin estimation was carried out by ELISA as per the manufacturer's protocol.

- Serum bilirubin was determined with standard procedure through a bilirubinometer.

- Sepsis screen was considered positive when any two of the following laboratory criteria were present: Total leucocyte count (TLC) $<5000 / \mathrm{mm}^{3}$, immature/total neutrophils ratio $\geq 0.2$, ANC $<1800$ and positive CRP (CRP $>10 \mathrm{mg} / \mathrm{dL}$, IL-6 positive (Value $\geq 20 \mathrm{pg} / \mathrm{mL}$ ), procalcitonin positive (value $\geq 10 \mathrm{pg} / \mathrm{mL}$ ).

- Moderate jaundice (total serum bilirubin $10-15 \mathrm{mg} / \mathrm{dL}$ ) and severe jaundice (total Serum bilirubin $>15 \mathrm{mg} / \mathrm{dL}$ )

\section{RESULTS}

During the study period, 170 neonates were included for confirmation of septicaemia. Out of the study subjects, $56.5 \%$ (96/170) were male and 43.5\% (74/170) were female. Age distribution of this study group showed $42.4 \%$ (72/170) had early onset sepsis and remaining 57.6\% (98/170) had features of late onset septicaemia depicted in Figure 1.

Most common perinatal risk factor was Premature Rupture of Membrane (PROM). Out of all study subjects, $18 \%$ (30/170) had history of PROM. Out of which $60 \%(18 / 30)$ developed sepsis, second common cause was preterm instrumentation, $54 \%(92 / 170)$ had more than three perinatal risk factors for sepsis as shown in Figure 2.

Serum bilirubin estimation shows out of 170 neonates, 110 were suffering from moderate (Serum bilirubin $10-15 \mathrm{mg} / \mathrm{dl}$ ) to severe jaundice (Serum bilirubin $>15$ $\mathrm{mg} / \mathrm{dL}$ ), of which $49 \%$ (54/110) were diagnosed to have sepsis, whereas in rest 56 neonates with mild or no jaundice (Serum bilirubin < $10 \mathrm{mg} / \mathrm{dL}), 14.3 \%(8 / 56)$ were diagnosed to have sepsis. 
Sepsis screen in the form of C-reactive protein $(>1.2$ $\mathrm{mg} / \mathrm{dL})$, TLC $(<5000$ or $>18000 / \mathrm{cu} \mathrm{mm})$, raised IT ratio ( $>$ $0.2)$, neutropaenia $(<1800)$, Micro-ESR were performed for all study subjects and $\geq 2$ parameter was found to be positive in $52.9 \%(90 / 170)$ cases.

Blood culture were done for those 90 cases showing sepsis screen positive, out of those $67.4 \%$ (62/90) came as positive by culture. Bacteriological profile of 62 culture positive cases shows $74.2 \%(46 / 62)$ and $25.8 \%$ (16/62) were suffering from Gram Negative Bacilli (GNB) and Gram Positive Cocci (GPC) infection respectively. Among GNB, Klebsiella spp. $43.5 \%(20 / 46)$ accounts for the most predominant organism causing neonatal sepsis followed by Escherichia coli 26.1\% (12/46). Among GPC, Staphylococcus aureus with $62.5 \%(10 / 16)$ accounts for the most frequently occurring pathogen causing neonatal sepsis which is followed by Streptococcus spp. 37.5\% (6/16) shown in Figure 3.

Antibiotic susceptibility pattern of GNB shows high degree of resistance towards penicillins (74\%), cephalosporins (37\%), fluoroquinolones (40\%) and tetracycline $(35 \%)$, whereas less degree of resistance was shown towards aminoglycosides (26\%), Chloramphenicol (20\%) and imipenem (0\%).

In cases of GPC, higher degree of resistance was shown towards penicillin $(75 \%)$ followed by Erythromycin (44\%) and Clindamycin (19\%), whereas Moxifloxacin (12.5\%), Vancomycin $(0 \%)$ and Linezolid $(0 \%)$ were showing very less incidence of resistance.

Among 62 confirmed neonatal sepsis case, mortality at the end of the study was found to be $41.9 \%(26 / 62)$.

Out of 62 blood culture positive cases, $64.5 \%(40 / 62)$ were IL-6 positive (Cut-off value $20 \mathrm{pg} / \mathrm{mL}$ ), whereas procalcitonin was positive (cut-off value $10 \mathrm{pg} / \mathrm{mL}$ ) among 95.1\% (59/62) cases.

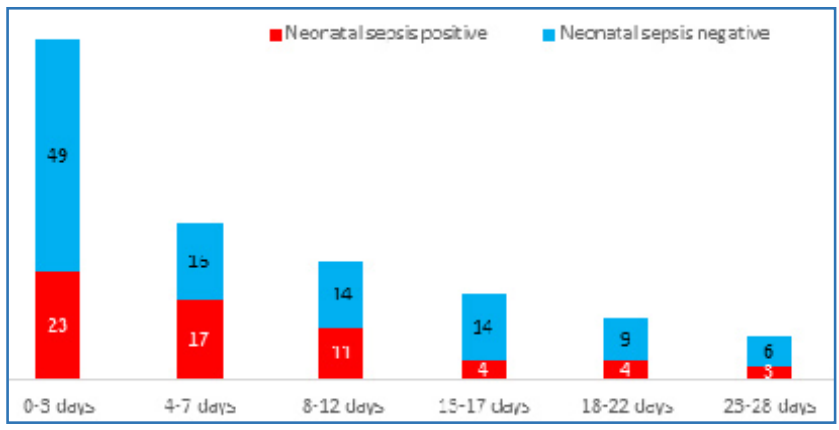

Figure 1. Age Distribution

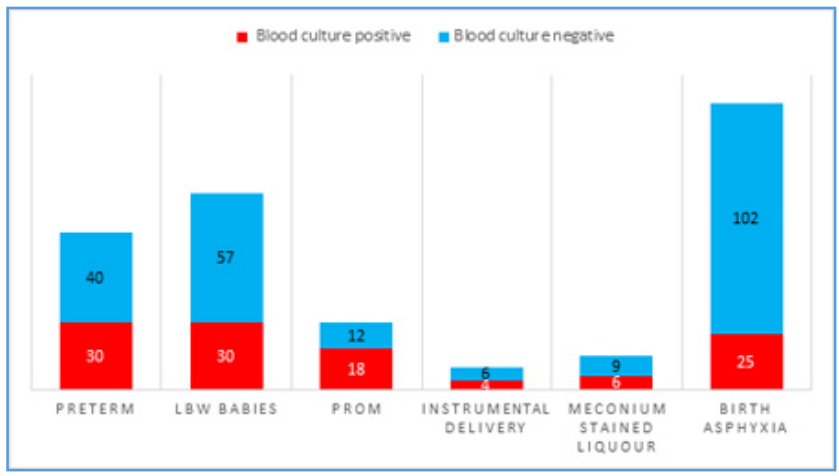

Figure 2. Distribution According to Maternal and Foetal Risk Factors

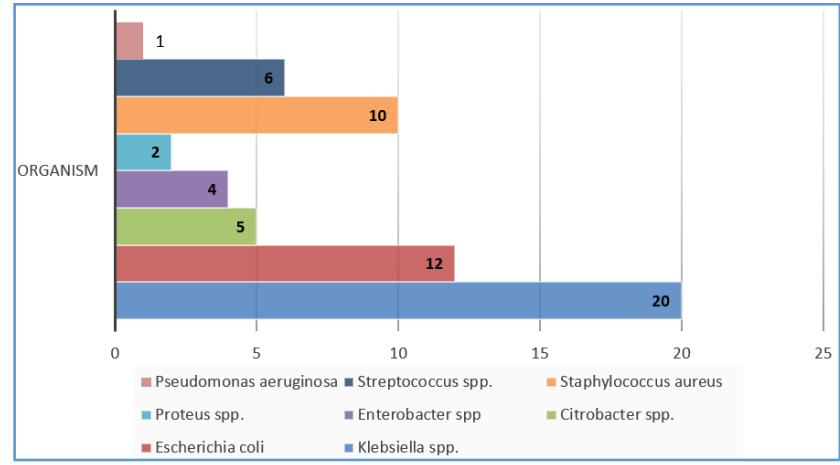

Figure 3. Distribution of Organism Isolated in the Study

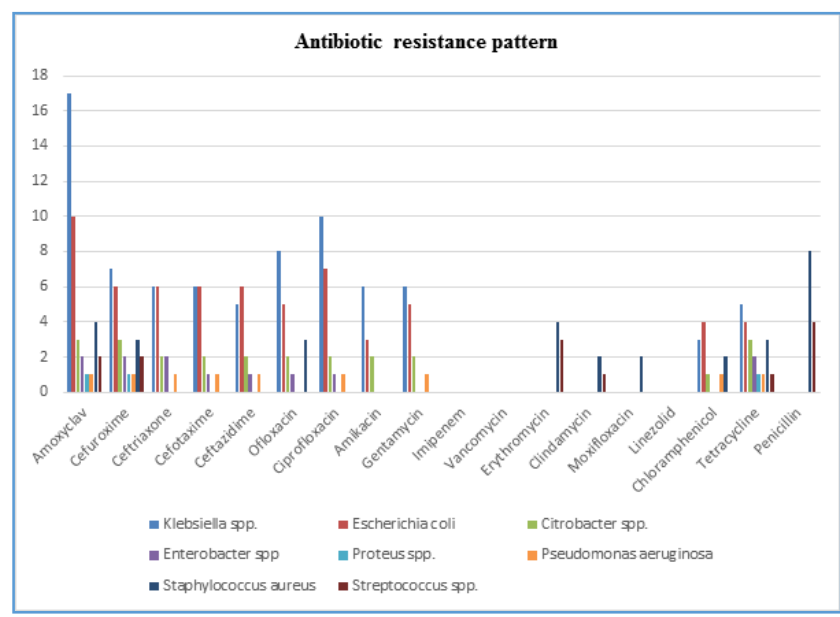

Figure 4. Antibiotic Resistance Pattern of Isolated Pathogens

\section{DISCUSSION AND CONCLUSION}

This study is the first of its kind undertaken in our State. The study shows slight predominance of male subjects over female, but occurrence of sepsis was similar in both sexes. Though 0 - 3 days' age group neonates represents bulk of study subjects; however, rate of LOS was higher in the study.

Among foetal risk factors prematurity and low birth weight were the most important factors associated with sepsis, whereas instrumental delivery and PROM were the most important maternal risk factor contributing to neonatal sepsis. It is in accordance with a study done by Mamta Jajoo, Kapil Kapoor and L K Garg et al. They found most common perinatal risk factor was LBW in $68 \%$ cases followed by PROM 56\%.[20]

Moderate-to-severe jaundice was an important marker of sepsis, which was reaffirmed in this present study where $49 \%$ of the neonates having moderate-to-severe jaundice had evidence of sepsis as compared to $14.3 \%$ of sepsis patient had mild or no jaundice. This study highlighted the superiority of procalcitonin over IL-6 as a screening tool for neonatal sepsis. Vazzalwar $\mathrm{R}$ et al, in a study also found that procalcitonin is superior.[15] In a study, Naher B S et al found sensitivity of $65 \%$ and Negative Predictive Value (NPV) (39.1\%) were higher for PCT, whereas specificity (90\%) and Positive Predictive Value (PPV) were lower in comparison to CRP.[19]

Sensitivity of PCT varied between $83 \%-100 \%$ and specificity varied between $70 \%-100 \%$. Junyan Qu et al also 
reported that PCT as a valuable marker of bacterial infections in febrile patients. PCT was superior to CRP, IL-6.[21] In a study conducted by Minoo Adib et al at Alzahra and Dr. Beheshti Hospital in and Fatema-Zahra in Najafabad from May 2005 to April 2006 found that PCT level was significantly higher in septic infants in comparison with the normal infants and it is $70 \%$ sensitive and $80 \%$ specific as a marker for the early diagnosis of neonatal sepsis.[22]

Bacteriological profile showed the predominance of Klebsiella spp. causing sepsis followed by Escherichia coli and Staphylococcus aureus. Among GNB high degree of resistance towards penicillins, cephalosporins, fluoroquinolones and tetracycline was observed and less degree of resistance was shown towards aminoglycosides, chloramphenicol and imipenem, whereas in case of GPC higher degree of resistance was shown towards penicillin followed by Erythromycin and Clindamycin, whereas Moxifloxacin, Vancomycin and Linezolid were showing very less incidence of resistance. It is in accordance with other studies.[23,24]

Though the current neonatal mortality rate of 44 per 1000 live births accounts for two-thirds of the infant mortality in India as per National Neonatal Prenatal Database.[5] However, our study shows mortality due to sepsis in $41.9 \%$; this could be due to late referral of the patients from peripheral health facilities, as it is the only tertiary care hospital of the state. Awareness among the health care providers and high degree of clinical suspicion may help in reducing the mortality due to sepsis.

In conclusion prevalence of neonatal sepsis was found to be high with presence of resistant phenotypes of pathogens in our setup. Proper implementation of Hospital Infection Control Practice and development of antibiotic stewardship program is the need of the hour. Detection of acute phase reactants like IL-6, Procalcitonin can predict in initiating appropriate treatment at the earliest where blood culture facilities are not available, which may help in judicious use of antibiotics.

\section{Acknowledgement}

The authors wish to thank the Indian Council of Medical Research (ICMR), New Delhi, India for support to carry out the study. Authors also thank all the staff of the project for their sincere help.

\section{REFERENCES}

1. Black RE, Cousens S, Johnson HL, et al. Global, regional, and national causes of child mortality in 2008: a systematic analysis. Lancet 2010;375(9730):1969-87.

2. Newton 0, English M. Young infant sepsis: aetiology, antibiotic susceptibility and clinical signs. Trans R Soc Trop Med Hyg 2007;101(10):959-66.

3. Bang AT, Bang RA, Baitule SB, et al. Effect of home-based neonatal care and management of sepsis on neonatal mortality: field trial in rural India. Lancet 1999;354(9194):1955-61.

4. Stoll BJ. The global impact of neonatal infection. Clin Perinatol 1997;24(1):1-21.

5. Report of the National Neonatal Perinatal Database (National Neonatology Forum) 2002-03.
6. Remington JS, Klein JO, Wilson $\mathrm{CB}$, et al. Infectious diseases of foetuses and new born infants. N Engl J Med 2006;355:531-2.

7. Panero A, Pacifico L, Rossi N, et al. Interleukin 6 in neonates with early and late onset infection. Paediatr Infect Dis J 1997;16(4):370-75.

8. Baier RJ, Loggins J, Yanamandra K. IL-10, IL-6 and CD14 polymorphisms and sepsis outcome in ventilated very low birth weight infants. BMC Med 2006;4:10.

9. Sastre LJB, Solis PD, Serradilla RV, et al. Procalcitonin is not sufficiently reliable to be the sole marker of neonatal sepsis of nosocomial origin. BMC Pediatr 2006;6:16.

10. Benitz WE. Adjunct laboratory tests in the diagnosis of early-onset neonatal sepsis. Clin Perinatol 2010;37(2):421-38.

11. van Rossum AM, Wulkan RW, Oudesluys-Murphy AM. Procalcitonin as an early marker of infection in neonates and children. Lancet Infect Dis 2004 Oct;4(10):620-30.

12. Santuz P, Soffiati M, Dorizzi RM, et al. Procalcitonin for the diagnosis of early-onset neonatal sepsis: a multilevel probabilistic approach. Clin Biochem 2008;41(1415):1150-55.

13. Mathers NJ, Pohlandt F. Diagnostic audit of C-reactive protein in neonatal infection. Eur J Pediatr 1987;146(2):147-51.

14. de Bont ES, Martens A, van Raan J, et al. Tumor necrosis factor-alpha, interleukin-1 beta and interleukin-6 plasma levels in neonatal sepsis. Pediatr Res 1993;33(4 Pt 1):380-3.

15. Vazzalwar R, Pina-Rodrigues E, Puppala BL, et al. Procalcitonin as a screening test for late-onset sepsis in preterm very low birth weight infants. J Perinatol 2005;25(6):397-402.

16. Kilpatrick L, Harris M. Cytokines and the inflammatory response. In: Polin R, Fox W. eds. Fetal and neonatal physiology. Philadelphia: WB Saunders Company 1998: 1967-79.

17. Whicher J, Bienvenu J, Monneret G. Procalcitonin as an acute phase marker. Ann Clin Biochem 2001;38(Pt 5):483-93.

18. Brunkhorst FM, Eberhard OK, Brunkhorst R. Discrimination of infectious and non-infectious causes of early acute respiratory distress syndrome by procalcitonin. Crit Care Med 1999;27(10):2172-6.

19. Naher BS, Mannan MA, Noor K, et al. Role of serum procalcitonin and C-Reactive Protein in the diagnosis of neonatal sepsis. Bangladesh Med Res Counc Bull 2011;37(2):40-6.

20. Jajoo M, Kapoor K, Garg LK, et al. Study of incidence and risk factors of early onset neonatal sepsis in an out born neonatal intensive care unit of India. Journal of Clinical Neonatology 2015;4(2):91-95.

21. Qu J, Lü X, Liu Y, et al. Evaluation of procalcitonin, Creactive protein, interleukin- $6 \&$ serum amyloid $A$ as diagnostic biomarkers of bacterial infection in febrile patients. Indian J Med Res 2015;141(3):315-21.

22. Adib M, Bakhshiani Z, Navaei F, et al. Procalcitonin: a reliable marker for the diagnosis of neonatal sepsis. Iran J Basic Med Sci 2012;15(2):777-82. 


\section{Jemds.com}

23. Kapoor L, Randhawa VS, Deb M. Microbiological profile of neonatal septicemia in a pediatric care hospital in Delhi. J Commun Dis 2005;37(3):227-32.

\section{Original Research Article}

24. Das PK, Basu K, Chakraborty P, et al. Clinical and bacteriological profile of neonatal infections in metropolitan city based medical college nursery. J Indian Med Assoc 1999;97(1):3-5. 